\title{
Micro-Hole and Strip Plate (MHSP) operation in $\mathrm{CF}_{4}$
}

\author{
H. Natal da Luz ${ }^{a}$, ,*, J.A. Mir ${ }^{\text {c }}$, J.F.C.A. Veloso ${ }^{a}$, J.M.F. dos Santos ${ }^{b}$, \\ N.J. Rhodes ${ }^{\mathrm{c}}$, E.M. Schooneveld ${ }^{\mathrm{c}}$ \\ ${ }^{a}$ Departmento de Física, Universidade de Aveiro, P-3810-193 Aveiro, Portugal \\ ${ }^{\mathrm{b}}$ Departmento de Física, Universidade de Coimbra, P-3004-516 Coimbra, Portugal \\ ${ }^{\mathrm{c}}$ CCLRC Rutherford Appleton Laboratory, Chilton, Didcot, Oxon, OX11 0QX, UK
}

Available online 18 May 2007

\begin{abstract}
The Micro-Hole and Strip Plate (MHSP) is a hybrid electron multiplier which combines the working principles of a Gas Electron Multiplier (GEM) and a Micro-Strip Gas Counter (MSGC). The compact double stage electron multiplication processes found in the MHSP enables the realisation of higher gas gain than the lone GEM operation. Thermal neutron detection using gas detectors involves the use of ${ }^{3} \mathrm{He}$ gas with another suitable stopping gas, operated at elevated pressure to confine the products of the neutron- ${ }^{3} \mathrm{He}$ reaction. It is, however, well known that the gain of GEMs drops too sharply with increasing chamber pressure.

We have pursued experimental work using the MHSP to infer the upper limits of thermal neutron position resolution. The stopping gas used during the present studies was Tetrafluoromethane $\left(\mathrm{CF}_{4}\right)$ chosen due to its low X-ray and $\gamma$-ray efficiency, requiring only 2.6 bar to yield a position resolution of $1 \mathrm{~mm}$. In the present studies, systematic measurements were taken to establish the gain versus $\mathrm{CF}_{4}$ pressure characteristics of the MHSP, in the 1 to 2.65 bar range. These studies demonstrated that it was possible to sustain gains above $10^{4}$ and 300 with pressures of 1 and 2.65 bar, respectively. The advantage of having two multiplication stages in the MHSP separated by only few tens of microns, results in a less pronounced gain reduction with pressure, presenting higher gains than single or multiple GEMs. The energy resolution at 1 bar was $29 \%$ FWHM for the $5.9 \mathrm{keV} \mathrm{Mn} \mathrm{K}$ line from a ${ }^{55} \mathrm{Fe}$ source, which is a typical value for micro-patterned gas detectors operating in $\mathrm{CF}_{4}$.
\end{abstract}

(C) 2007 Elsevier B.V. All rights reserved.

PACS: 29.40.Cs; 29.40.Gx; 85.60.Gz

Keywords: Micro-hole and strip plate; Thermal neutrons; Effective gain

\section{Introduction}

The Micro-Hole and Strip Plate (MHSP) [1] is a hybrid detector combining a Micro Strip Gas Chamber (MSGC) [2] and a Gas Electron Multiplier (GEM) [3] in opposite sides of the same substrate, having the advantage of two multiplication stages. The MHSP has been intensely investigated, showing very good detection capabilities, providing high gains and good energy resolutions when compared to other micro-pattern devices, including its operation under high pressure [4,5]. Imaging capability can be achieved by reading the anodes either individually or in

\footnotetext{
${ }^{*}$ Corresponding author. Tel.: + 351234378 108; fax: + 351234424965 .

E-mail address: hugo@gian.fis.uc.pt (H. Natal da Luz).
}

groups on the MSGC side, for one dimension and structuring the GEM side for the second dimension.

The aim of this work is to infer the ability of the MHSP for thermal neutron detection and evaluating an upper position resolution limit using ${ }^{3} \mathrm{He} / \mathrm{CF}_{4}$ mixtures. From several possible nuclear reactions for neutron capture and subsequent detection, one of the most common is the reaction of thermal neutron with ${ }^{3} \mathrm{He}$ nucleus yielding a $573 \mathrm{keV}$ proton and a $192 \mathrm{keV}$ tritium: ${ }^{3} \mathrm{He}(\mathrm{n}, \mathrm{p}) \mathrm{T}$.

For position detection capability, the centre of mass of the avalanches originated by the proton and the tritium is measured, which means that there is always a systematic error associated with the measurement. This error is dependent on the range of the two particles in the medium. The range of $573 \mathrm{keV}$ protons in ${ }^{3} \mathrm{He}$ at 1 bar is too long [6], which imposes the need for a high pressure operation as 
well as the inclusion of a suitable stopping gas to restrict the proton range to approximately $1.4 \mathrm{~mm}$. $\mathrm{CF}_{4}$ is used for this purpose, due to its low $\mathrm{X}$-ray and gamma ray efficiency. Furthermore, when using $\mathrm{CF}_{4}$, a modest pressure of only 2.6 bar is required to stop the protons within $1.2 \mathrm{~mm} \mathrm{[6].}$

The minimum gain required for thermal neutron detection is 10 , taking into account the electronics for signal acquisition. It is well known that the gain of conventional GEM drops very sharply with increasing stopping gas pressures [7]. In addition, the GEMs operation at elevated pressure becomes limited due to the maximum voltage being reached before the onset of discharge processes. For example, a single GEM operating in $\mathrm{CF}_{4}$ achieves a gain of only about 3 at 3 bar [8]. Recent work involving operation of the MHSP with noble gases at elevated pressures, however, has shown the possibility of much higher gains than either a single GEM or triple GEMs $[5,9,10]$. We have therefore foreseen distinct advantages in using the MHSP for thermal neutron detection and carried out a systematic investigation on the operation of the MHSP in $\mathrm{CF}_{4}$, in the 1 to $2.65 \mathrm{bar}$ range.

\section{Experimental set-up}

The MHSP used for this study consists of a $50 \mu \mathrm{m}$ kapton foil substrate, where a copper layer of $5 \mu \mathrm{m}$ thickness was deposited on both sides and patterned as a GEM in one side and as a microstrip geometry with the holes emerging from the cathodes on the other side. The microstrip pattern has a pitch of $200 \mu \mathrm{m}$ with width of the anodes and cathodes set at $20 \mu \mathrm{m}$ and $100 \mu \mathrm{m}$, respectively. The $60 \mu \mathrm{m}$ diameter holes have a distance of $140 \mu \mathrm{m}$ between their centres along the cathode and $200 \mu \mathrm{m}$ in the orthogonal direction. The detector has a $25 \mu \mathrm{m}$ mylar window and a drift region of $5 \mathrm{~mm}$. The drift field used was $40 \mathrm{~V} / \mathrm{cm}\left(V_{\mathrm{T}} / 0.5\right)$. This field was not optimised for energy resolution. For the measurements, $5.9 \mathrm{keV} \mathrm{Mn}$ and $22.1 \mathrm{keV} \mathrm{Ag} \mathrm{X-rays} \mathrm{were} \mathrm{used} \mathrm{to} \mathrm{study} \mathrm{the} \mathrm{detector}$ performance with $\mathrm{CF}_{4}$ pressures up to 2.65 bar.

The charge pulses from the detector were collected by a Canberra 2006 charge pre-amplifier with a charge sensitivity of $235 \mathrm{mV} /$ Mion-pair and then shaped by a Tenelec TC243 amplifier with a shaping time of $4 \mu$ s. To establish a relationship between the pulse height and the detector gain, a calibrated $2 \mathrm{pF}$ capacitor was used to convert the voltage signal from a BNC pulse generator and provide a well known charge signal to the pre-amplifier input. Having the information of the $W$ value for $\mathrm{CF}_{4}$, the number of primary electrons per X-ray photon is known and the detector gain can then be determined.

\section{Measurements and discussion}

Fig. 1 shows the variation of the effective gain (M) as a function of the voltage applied across the MHSP holes

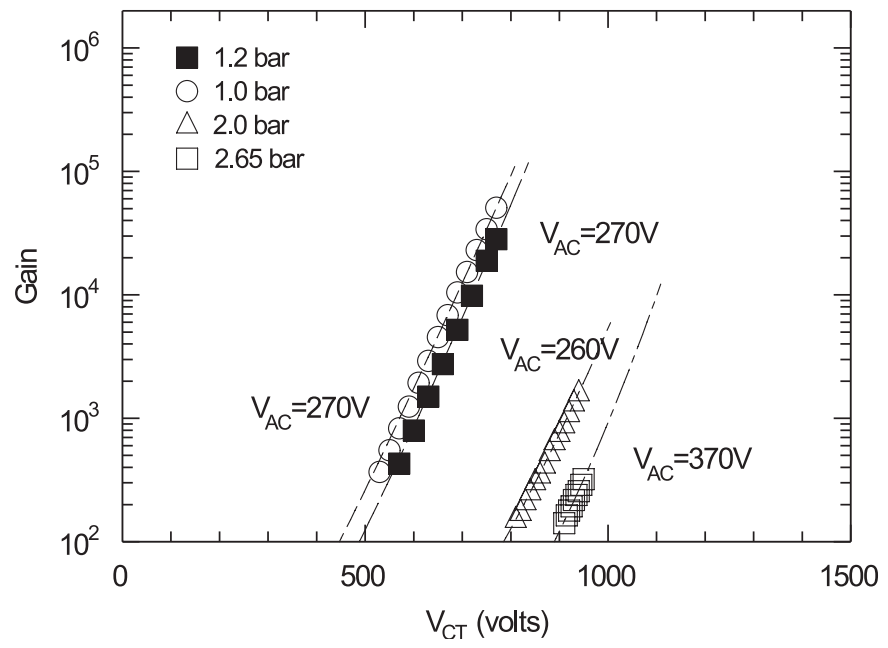

Fig. 1. Gains obtained with MHSP operating in $\mathrm{CF}_{4}$ as a function of applied voltage for a number of different pressures. VAC is the voltage between anodes and cathodes on the strip-side.

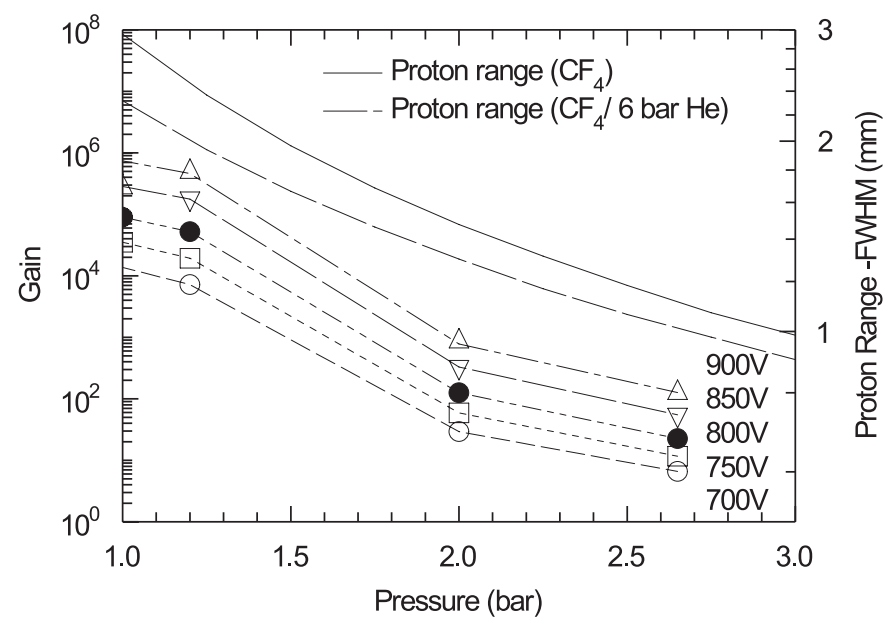

Fig. 2. Variation of MHSP gain and calculated position resolution (for $573 \mathrm{keV}$ protons) with applied $\mathrm{CF}_{4}$ pressure for a number of different applied voltages. Note the additional curve showing the expected position resolution if 6 bar helium is added to a given $\mathrm{CF}_{4}$ pressure.

$\left(V_{\mathrm{CT}}\right)$ for a number of different $\mathrm{CF}_{4}$ pressures. Fig. 1 also shows curve fits using a generalised gain formula [11]:

$\ln (M)=\left(V_{\mathrm{CT}} / a\right) \exp \left(b /-V_{\mathrm{CT}}\right)$

where $a$ and $b$ are constants. The higher values of the gain shown in Fig. 1 correspond to the upper voltages before the onset of discharges. It was observed that the gains obtained with the MHSP operating in $\mathrm{CF}_{4}$ are well above the required value of 10 for thermal neutron detection. Using the fitted data shown in Fig. 1, it was possible to plot gain as a function of pressure for given $V_{\mathrm{CT}}$ values as shown in Fig. 2. The achievable detector gain decreases with pressure as expected. However, this decrease is not as pronounced as in other microstructures such as the conventional GEM [8]. We were able to demonstrate stable operation of the 


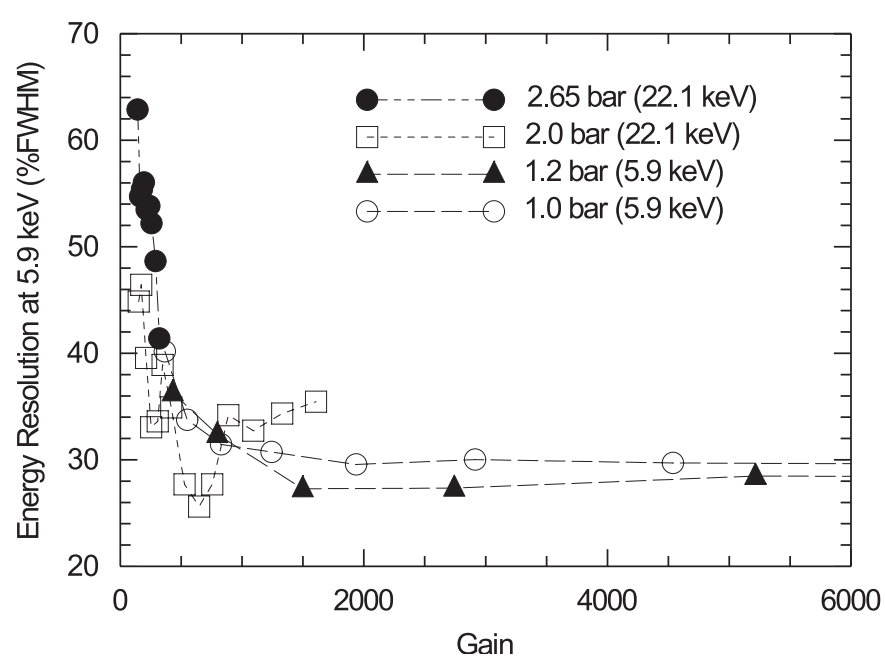

Fig. 3. X-ray energy resolution (\%FWHM) of the Mn K X-rays $(5.89 \mathrm{keV})$ for $\mathrm{CF}_{4}$ pressures of 1 and 1.2 bar and $\mathrm{Ag} \mathrm{K}$ X-rays $(22.1 \mathrm{keV})$ for $\mathrm{CF}_{4}$ pressures of 2 and 2.65 bar as a function of the MHSP gain. In all cases the drift potential $V_{\mathrm{T}}$ was maintained at $20 \mathrm{~V}$.

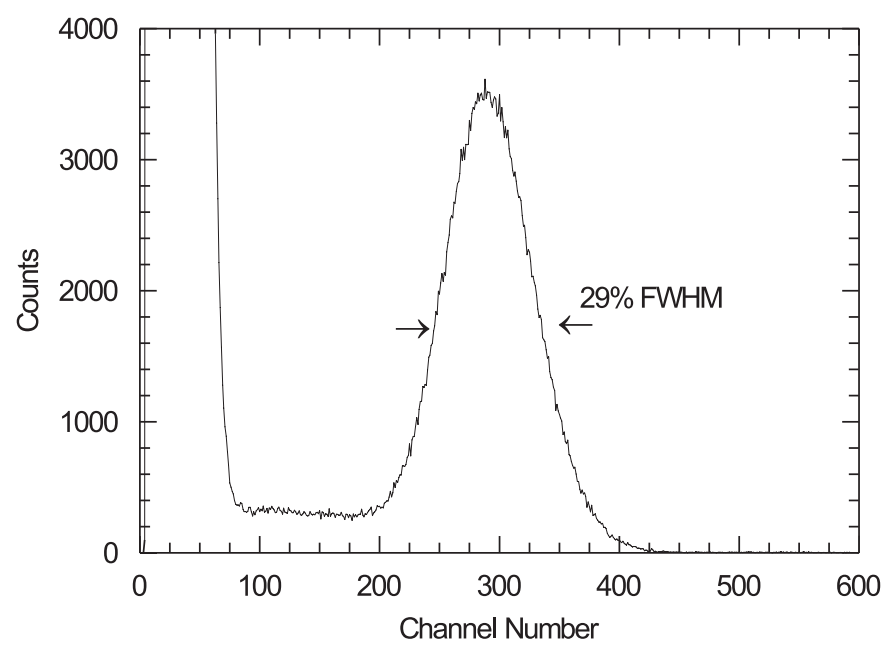

Fig. 4. Pulse height Spectrum of Mn K X-ray line (5.9 keV) from Fe-55 source with $\mathrm{CF}_{4}$ at 1 bar. MHSP was held at gain of 1000 with, $V_{\mathrm{CT}}$ and $V_{\mathrm{AC}}$ at 510 and $290 \mathrm{~V}$, respectively.

present MHSP over the gain of 300 using $\mathrm{CF}_{4}$ at a pressure of 2.65 bar.

Fig. 3 shows the variation of the X-ray energy resolution as a function of the MHSP gain, when $\mathrm{CF}_{4}$ pressure was set at $2.65,2.0,1.2$ and 1 bar. The optimum resolution occurred when the MHSP gain exceeded a value of 1000 , being approximately $29 \% \mathrm{FWHM}$ at $5.9 \mathrm{keV}$ and therefore poorer than with our previous studies on the MHSP albeit using noble gas mixtures [12-14]. Fig. 4 is a spectrum obtained with the MHSP operating at a gain of around 1000 with $\mathrm{CF}_{4}$ at 1 bar. As can be seen from Fig. 4, the photopeak was completely separated from the detector noise located below $\sim 2 \mathrm{keV}$.

\section{Conclusion}

We observed that although the MHSP gain decreased with increasing $\mathrm{CF}_{4}$ pressure, it was not as pronounced as with other microstructures, such as previous GEM based studies $[8,15]$. Our experimental work showed a stable operation of the MHSP over the gain of 300 using $\mathrm{CF}_{4}$ at 2.65 bar. These studies therefore successfully demonstrated that it will be possible to detect thermal neutrons whilst achieving a spatial resolution at $1 \mathrm{~mm}$ or better provided one advocates the use of a more advanced microstructure such as the MHSP. Further studies will be made in the near future, with a gas mixture of 6 bar ${ }^{3} \mathrm{He}$ and 2.6 bar $\mathrm{CF}_{4}$.

\section{Acknowledgements}

Support is acknowledged from project POCTI/FNU/ 50360/02 through FEDER and FCT (Lisbon). H. Natal da Luz acknowledges grant SFRH/BD/16342/04 from FCT.

\section{References}

[1] J.F.C.A. Veloso, J.M.F. dos Santos, C.A.N. Conde, Rev. Sci. Instr. 71 (6) (2000) 2371.

[2] A. Oed, Nucl. Instr. and Meth. A 263 (1988) 351.

[3] F. Sauli, Nucl. Instr. and Meth. A 386 (1997) 531.

[4] J.F.C.A. Veloso, et al., IEEE Trans. Nucl. Sci. NS-51 (5) (2004) 2104.

[5] F.D. Amaro, et al., J. Inst. 1 (2006) P04003.

[6] J.F. Ziegler, J.P. Biersack, SRIM-The Stopping and Range of Ions in Matter, 〈URL: http://www.srim.org//, 2003.

[7] A. Breskin, A. Buzulutskov, R. Chechik, Nucl. Instr. and Meth. A 483 (2002) 670.

[8] R. Kreuger, et al., IEEE Trans. Nucl. Sci. NS-51 (5) (2004) 2104.

[9] A. Bondar, et al., Nucl. Instr and Meth. A 419 (1998) 418.

[10] A. Buzulutskov, Nucl. Instr. and Meth. A 494 (2002) 148.

[11] J.E. Bateman, Phys. Rep. 375 (6) (2003) 411.

[12] F. Amaro, et al., Nucl. Instr. and Meth. A 535 (2004) 341.

[13] J.F.C.A. Veloso, et al., Nucl. Instr. and Meth. A 524 (2004) 124.

[14] J.M. Maia, et al., IEEE Trans. Nucl. Sci. NS-49 (3) (2002) 875 (pt. 2).

[15] A. Kozlov, et al., Nucl. Instr. and Meth. A 524 (2004) 345. 